\title{
Looking for Culture in Video Games: Three Conceptual Approaches
}

\author{
James K. Scarborough ${ }^{*}$ \\ Department of Communication, Stanford University, \\ McClatchy Hall, 450 Serra Mall, Stanford, CA 94305, USA \\ joseppi@stanford.edu
}

\begin{abstract}
As the popularity of computer games has increased markedly in recent years, many researchers have become concerned with the potential effects on gamers. However, by looking past the outward appearance of popular games that may be perceived as violent by some, we can begin to see dramatic cultural indicators as well as many other academically interesting phenomena emerging. Social psychologists and effects researchers must begin delving deeper into this rapidly expanding genre of popular media. Herein, I propose three possible approaches to the study of video games as cultural events rather than a common medium that influences participants in a simple way.
\end{abstract}

Keywords: Video game, culture, play, media effects, social influence, psychology, research methods.

\section{The Culture of Modern Media}

In the beginning there was radio. And it was good. We gathered on Saturday nights to listen to stories, news, and music. Radio brought us together in a way that only government and religion had done before then. Not only was the entire family sitting around the monophonic radio receiver box at the same time, the entire nation was present. Together we shared experiences that quickly became cultural events with their own rules, norms, and traditions. Don't be late or you'll miss the best part of the program, 8 o'clock sharp! Yet we craved interesting imagery and visual stimulation. We wanted to see what was happening.

Then there was television. TV in all its audio-visual glory: black and white maybe but to us as vivid as real life. We were the family living in suburban America, with two kids and a family car. Again, we quickly established our scheduled traditions and program preferences. If little Johnny didn't finish his homework he wasn't going to be allowed to participate in the ritual which, of course, was socially unacceptable among his school friends. However, whether we were cheering our favorite sports team on to victory or we were hootin' and hollerin' with the Lone Ranger and Tonto, we longed to be part of the action. We became so intimate with the characters of our favorite soap operas that we just wanted to reach out and tell them how we felt about

\footnotetext{
${ }^{*}$ Corresponding author.
} 
their recent actions or comfort them in their time of need. But television couldn't hear us. Television only told and never listened.

So came the Internet, the Web, web 2.0, and we were heard. A new form of news, entertainment, and information gathering was at our very fingertips. We found others, nay many others who were also fans of old-time radio programs like The Shadow. Not only did they share their thoughts and insights about the program but recordings of the programs were made available for us to enjoy all over again. We regained the nearly lost sense of community that the potential personalization of the Internet almost destroyed. Once again, we belonged. We quickly came to know the rules, norms, and traditions of our newfound communities or risked being flamed and marked as a newbie. But alas, as major businesses, educational institutions, and masses of social groups came together, the form of their community was merely text and images. So much data yet so few tools to receive, compose, and transmit. Visual and auditory, yes, interactive maybe, but "real-time" still a long way off; nowhere near as good as being there.

\subsection{Video Games as Modern Media}

Arguably, video games are the only media able to meet the interaction needs of humans. Video games are audibly involving with amazing sound-tracks and sophisticated audible queues, visually exciting with ever-improving graphics and realism rivaling any other virtual reality technology. They are stimulatingly interactive with advanced artificial intelligence and intellectual challenges for even the most creative imagination, and inescapably real-time; turn away for one second and things can go seriously wrong for your avatar.

Even though video games are one of the most complex and sophisticated forms of modern media, the question remains: why study games? The fact that there are now more than 13 million World of Warcraft subscribers (a larger population than Sweden, Hungary, Cuba, or Greece) is interesting but seems more of value as a marketing statistic than cultural phenomenon. However, with the rapid advance and massive user populations of social/entertainment video games, clear indicators of emergent culture are starting to appear [cf. $6,7,15]$.

\subsection{A Few Important Distinctions}

At this point it is important to identify the taxonomy of video games. A great distinction exists between Pong and Pac-Man not to mention the modern Massively Multiplayer Online Game (MMOG for short) and the many other types of games, simulations, and virtual environments. Figure 1 shows an updated list of video game

- Arcade Style Action

- Action Adventure

- Construction and Management Simulation

- Role-playing

- Real-Time Strategy (RTS)

- Other notable genres: Music, Party, Puzzle, Sports
- First Person Shooter (FPS)

- Adventure

- Life Simulation

- Strategy

- Vehicle Simulation

Fig. 1. Modern Video Game Genres 
Achiever - Focus on achievement and material gain

Explorer - Focus on exploration and geographical knowledge

Socializer - Focus on socialization and making friends

Killer - Focus on engaging and dominating other players

Fig. 2.1. The Bartle Test of Gamer Psychology - Four Categories of Player

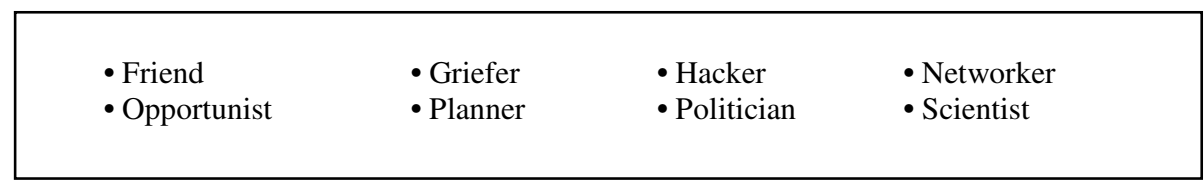

Fig. 2.2. Updated list of eight Gamer Categories

genres, originally and brilliantly conceived by Cris Crawford back in 1982 at the inception of the modern video game industry. One additional type of application that should be included in the list is the Multi-User Virtual Environment (MUVE). Since they typically lack any interactive game content or AI, MUVEs can not be strictly described as video games thus they are omitted from the list.

In addition, it is imperative to distinguish between several types of video gamer players. Figure 2.1 shows the four categories of computer gamer as first conceived by Richard Bartle in his Test of Gamer Psychology [4]. Figure 2.2 shows an updated list of categories based on feedback since Bartle's original Test was released. The Bartle Test has been taken by well over 500,000 gamers and works something like the Meyers-Briggs Personality Type Indicator. The answers to a series of questions lead to a type indicator result that describes the player's preferences and to some degree motivations. While the Bartle Test applies primarily to MMOG players, it identifies the myriad of game player personalities that contribute to the complexities of social interaction within game worlds.

\section{The Culture of Video Games}

In identifying, investigating, and interpreting video games as culture there are a great variety of ways to approach the research [7, 15]. Herein I will begin with just a few simple examples. We might consider video games as cultural events similar to sporting activities. With this approach we would need to stratify competitive game events by level of competition starting with casual local and regional competitions (a.k.a. LAN parties) and extending to major national events like the Korean Starcraft Championships that take place in giant stadium environments. Next we might attempt to assess the impact of the rapidly increasing number of computer gamers and the powerful sub-culture's influence on markets and cultures outside their traditions [6]. This approach would require a solid detailed knowledge of economics as well as a deep understanding of actual game mechanics that could potentially impact external forces. Finally, we might consider an ethnographic approach to video game players and the 
social institutions that are vital to coordinating complex interactions and events at the highest levels in MMOGs [15]. Any of these approaches as well as a variety of quantitative methods could be leveraged to yield very interesting research studies.

\subsection{Distinguishing Game from Play}

Before moving on to further details of the possible research frameworks, it will be beneficial to distinguish between game and play, a difference that is quite present in most all video games. This contrast differentiates the concepts of playing for fun and playing to win. Making this distinction also clarifies the divergence between video game genres as well as types of video gamers. Understanding this diversity will help explain the significance of different levels of video game competition, will assist in identifying the cultural impact of video games, and will support the existence and implications of social institutions and emergent culture in virtual worlds.

Most cultures have easily accessible examples of games. International sports are one example of game with which most of us are quite familiar. Take, for instance, the example of soccer or 'futbol'. Soccer is played in nearly every civilized country in the modern world. All you need is a ball, some friends, and a place to play. However, soccer is also the most popular competitive international sport in the modern world. While little children may play soccer in the street for fun with few consequences, serious athletes approach professional soccer as though each game was an epic battle for life or death between their team and "the enemy" or opposing team. There is no concept of play in professional sports. Teams are run as businesses, players are assets (or liabilities), and fans treat each win and loss as a personal victory or defeat in the quest for their team's greatness and a shot at a championship ring or trophy.

Similarly, the concept of play can be exercised both within and without the presence of a game. While children, of course, play sports they also play with blocks, play Cowboys and Indians, and play House. Games usually have distinct goals and outcomes (i.e. winning and losing or a final score) and games typically have a start and an end while play requires none of these things. Play might be defined simply as engagement in an activity with few or no consequences. With this definition of play it becomes clear that even Little League Baseball or Pop Warner Football games are not strictly for play.

One reason this distinction is relevant for studying video games is that popular belief suggests video games are purely for entertainment or distraction. This is certainly not the case when it comes to the more sophisticated games like MMOGs [13]. While there are many gamers who play MMOGs exclusively for entertainment, the vast majority of players who spend significant amounts of time with their avatars (20 hours per week) [16] have long since made fun a secondary pursuit. These players have far more diverse motivations. Gaining levels, building virtual wealth [6], establishing and maintaining social relationships [17], gaining knowledge of game mechanics and social norms, engaging in compelling narrative, building a reputation, and working toward common goals and objectives with friends and 'guildmates' are all far more typical motivations than simply playing for fun [17]. The reality of massive multiplayer games is that it is the minority who play for fun, the rest approach the activity more like professional athletes looking for better and faster ways to "win." This is also true, to a lesser extent, of nearly all competitive video games. 
Among high school gamers, anecdotal evidence suggests that a person's entire social reputation can be based on their ability to dominate other players in the most popular FPS games. Entire social enclaves are formed and might also be dissolved over the group's collective ability to consistently defeat other players within the game world. The most surprising aspect of this phenomenon is that the social groups that are formed ignore other common social norms and boundaries such as age, race, and even gender. If a player has the ability to contribute to the team's overall success then they will quickly be initiated and absorbed into the group for fear that an opposing team will recruit that player first.

This approach to competitive team building is paralleled, again, by major league sports [12]. Lewis suggests that traditional methods of valuing baseball players such as batting average or stolen bases, distinctly individual measures, are less effective than more modern statistical methods of valuation such as on-base percentage and slugging percentage which are more aligned with team performance than strictly individual statistics (i.e., Sabermetrics). Admittedly, the methods employed by most competitive video gamers are not quite as sophisticated as those described by Lewis. Yet their approach to team building by finding undervalued players goes against the more traditional method of finding the best individual players and recruiting them with teamwork as an afterthought.

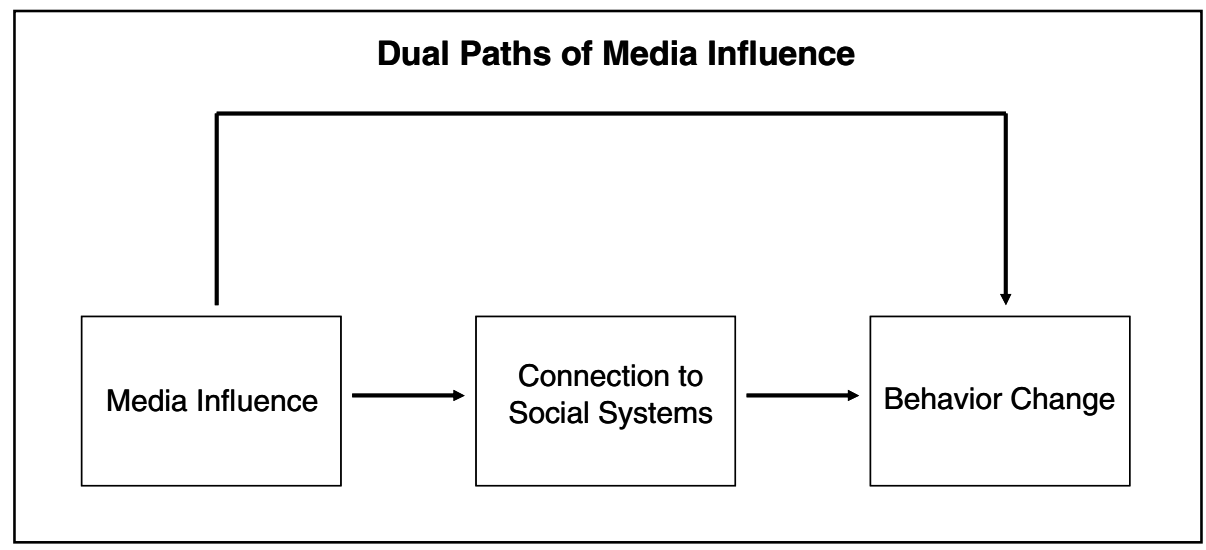

Fig. 3. Bandura's Dual Paths of Media Influence Model

\section{Implications for Research}

With these important distinctions in mind, it is now possible to move deeper into the task of finding the true nature of culture in video games. James Carey suggests that one way to go beyond the simple effects model of media is to change our approach from one of media consumption (the transportation model of media) to one of media as ritual [5]. This approach allows us to leverage the distinctions of media uses and gratifications [14] rather than rely on a naïve hypodermic model approach wherein media is studied as though it were a novel medication. By individuating players by type, a la Bartle, rather than aggregating all video game players as a group we can 
also begin to better understand the persuasive effects of gaming and involvement in gaming communities through more sophisticated means such as Bandura's Dual Path of Media Influence Model (see Figure 3). Using these methodologies to study both video gamers and the social institutions that drive high-end gaming, it becomes possible to move beyond the stigma that typically accompanies the study of video games. Researchers can now utilize the appropriate tools to study video games in a far more sophisticated way rather than the shallow superficial structure that is frequently engaged in existing research.

As video games are played in many different countries it will also be possible to differentiate the impact of games within geographically separate populations. There are two distinct issues at stake. First, there are the differences various cultures may have regarding types of games played. Clear differences in genre preference may help researchers to better understand the uses and gratifications of interactive media within that culture or may point to varying motivations for play. Second, it will be of interest to identify the variability in external impact of gaming on different cultures. While gamers in western countries may experience a high degree of social interaction playing certain types of games it is likely that gamers from other regions separated by language and cultural differences will have sharply contrasting experiences. Identifying these differences will ultimately lead to a much better understanding of racial issues that may be unwittingly perpetuated by the largely western dominated video game industry. Once the underlying issues are revealed it will be possible to address them through improved software and hardware design.

Geertz claims that the best way to understand a culture is to get involved by means of naturalistic observation [9]. Subsequently, researchers should rely on interpretation and 'thick description' to report findings. This framework allows research to provide a narrative of events and identify underlying meaning that even the actors involved may not fully understand. Attempting to arrive at a deeper understanding of a culture through standardized surveys and quantitative measures may well mislead a researcher and cause false conclusions to be made. This is the primary reason for many of the current myths surrounding video games. By aggregating members of the video game community and not differentiating between players who spend their time primarily in single-player games and those who spend their play time closely involved in active social institutions, resulting relationships can not realistically claim validity.

With this in mind, studies of game players must be differentiated by game genre as well as by player type and cultural affiliation. Even then, a variety of methodologies as well as a number of different research tools must be used to discover the deeper meaning of involvement in game communities $[7,15]$. The complexities and intricacies of MMOGs alone would warrant this not to mention the idiosyncrasies of mediated social relationships. When discussing doing research in the most popular MMOG to date, World of Warcraft, Corneliussen and Rettberg echo this sentiment by saying, "in order to understand World of Warcraft we must study it both as a game and as a cultural site requiring the application of multiple disciplines' analytical tools, concepts, and methods so that we may fully comprehend this phenomenon." 


\section{Three Conceptual Approaches}

\subsection{Simple Pastime or National Sport?}

In China they play table tennis, in America football, baseball, or basketball. In Korea they play Starcraft. In fact, there are several "professional" competitive Starcraft teams in Korea that boast major corporate sponsors, player's annual incomes that rival any American professional athlete, and multiple television channels providing complete tournament coverage.

The computer game StarCraft has an active professional competition circuit, particularly in South Korea. The two major game channels in South Korea, Ongamenet and MBCGame, each run a Starleague, viewed by millions of fans. Starting in about 2002, pro-gamers started to become organized into teams, sponsored by large South Korean companies like Samsung, SK Telecom and KTF. StarCraft is also the most popular computer game competition during the annual World Cyber Games thanks to its Korean fanbase, and it is overall one of the world's largest computer and video game competitions in terms of prize money, global coverage and participants. -Wikipedia

The real-time strategy video game Starcraft is not a new game. It was published in 1998 as "just another RTS" in a long line of successful games. However, the RTS player community did not accept this premise. As with many other aspects of the computer game industry, the players decided to take control of the game and change the rules [11].

Many publishers now provide players with an engine for generating game content. In the case of Starcraft, the generation engine allowed players to not only create new game maps but it allowed them to modify the fundamental rules of the game. With this new ability, players became level designers and co-authors of the game. Due to

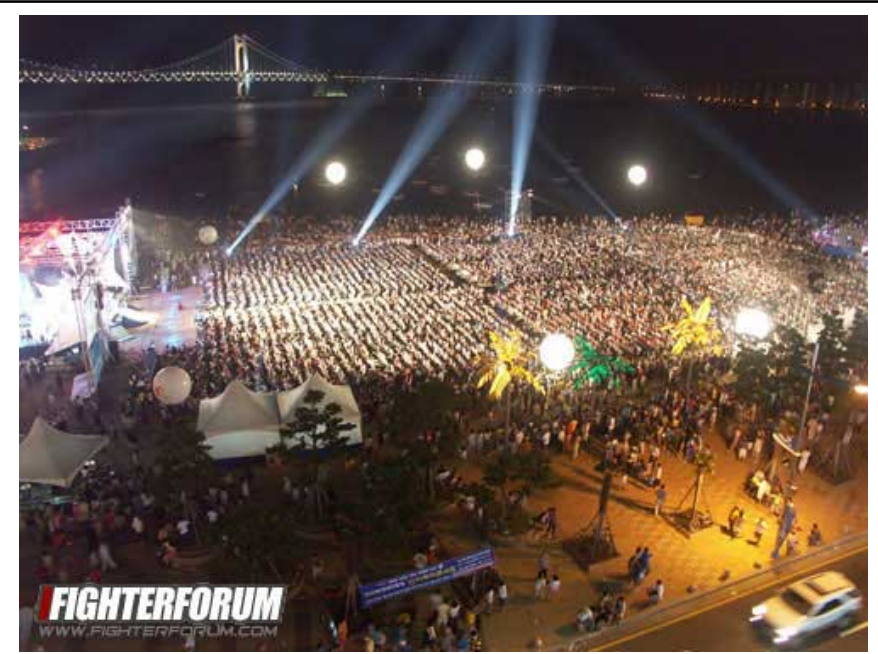

Fig. 4. An e-sports stadium where professional Starcraft games are played 
the popularity of the Starcraft map editor, the game publisher followed up with another more powerful system with the release of the game Warcraft III, arguably the most advanced RTS game ever made. Players generated new versions of game maps, new rule sets, and eventually a new game almost entirely different than the original Warcraft III known as Defense of the Ancients or DotA (pronounced Doe-ta). This series of events started a new trend in game publication that put players in control of the growth and transformation of new games. While this transition occurred over several years it altered the video game industry forever.

The competitions involving these games are played in stadium-sized venues and draw exceptionally large crowds as well as international press coverage (see Figure 4). This provides an excellent opportunity for study. Starcraft champions are considered national heroes in Korea the same way that professional athletes are treated in America. Another aspect of this phenomenon is how amateur Starcraft players are influenced by professional players. A small local competition can quickly be transformed into an international cultural event with the presence of a few professional players and the power of digital press coverage. Closer examination of this could reveal many interesting indications of how the new digital generation influences existing Korean culture and perhaps how the Korean gaming culture exerts influence on the rest of the world.

\subsection{Virtual Dollars to Real Dollars}

In 2005, Edward Castronova authored one of the earliest books that investigated video games' influence on the world outside the game [6]. While he mainly focused on economics he also explored some aspects of computer game player culture. The main theory put forward and backed up by solid data is that virtual economies found inside game worlds have a measurable influence on the real economy. This effect is due to two factors.

The first factor is the sheer number of players that are involved in games like World of Warcraft. In early 2009 over 13 million players subscribed to World of Warcraft. At around $\$ 15$ per month each, this number alone is economically significant totaling $\$ 2.3$ billion spent per year for a single game, not to mention the $\$ 30$ or $\$ 40$ initial cost per game license [8]. Granted, some number of subscribers may receive discounts and perhaps even earn free play time by volunteering to be a "guide" and helping other players from time to time with technical issues. Regardless, there is no arguing this dollar amount is respectable for any single software business.

The money spent by players to play the game is not the most interesting factor. Even more interestingly, the players or residents of virtual worlds including Second Life and World of Warcraft have begun spending large sums of money to acquire ingame virtual assets. Castronova followed the e-bay market sales of virtual game assets for a period of time. He established that, "The commerce flow generated by people buying and selling money and other virtual items (that is, magic wands, spaceships, armor) amounts to at least \$30 million annually in the United States, and \$100 million globally" [6]. While this may seem like a relatively small amount, the total has continued to grow rapidly over the years and while there is no more recent data on the trend, it is surely much higher today. Figure 5 shows a recent snap-shot of the actual exchange rates of Second Life's Linden dollars to real US dollars. Again, while the 


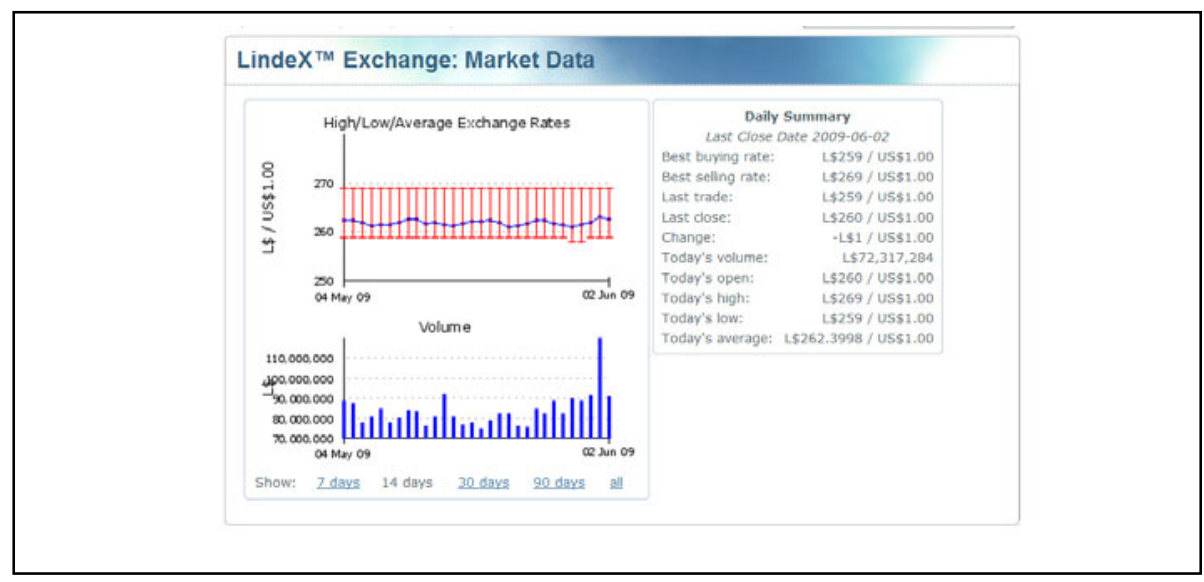

Fig. 5. Real market exchange rate data for Second Life's Linden dollars

exchange rate is not tremendous, the fact that a number of people assign real value to virtual items and currency gives those virtual items real value. As the popularity of MMOGs continues to increase, as all indicators suggest it will, the significance of this virtual to real exchange rate will become a sizeable market force.

In addition to the market influence of virtual worlds, Castronova also began exploring the real influence that virtual institutions such as player guilds can exert on real world social interaction. Beyond the anecdotal evidence of virtual relationships leading to real marriage, in-game groups have extended their interactions to the real world in the form of player meetings, fan faires, and conventions. These player summits often include game designers and corporate public relations. One of the objects in these circumstances is to hold meaningful discussions both among players and between players and game designers in an attempt to improve game mechanics and rules. Many game publishers are now taking fan faires very seriously and providing sponsorship and organization to enhance the players' out-of-game experience with the hopes it will positively affect their in-game experience as well.

\subsection{Players vs. Designers}

One of the most interesting trends in modern video games is the tendency of players to reverse their traditional role as consumers of the media. In the past, a game designer would create a game and the player would play it. This has been true for decades since the earliest video games like Pong and Venture (mainframe text game). Things have changed dramatically over the past decade. In order to increase the lifespan of their products as well as to meet the demands of an increasingly sophisticated player audience, many game designers now include "level editors" as a standard feature of their game. Level editors allow players to become designers by providing the ability to design custom game maps, deviant rule sets, and extended game play with increased complexity and difficulty. The original intention of level editors has indeed worked out to the designer's benefit helping games like Starcraft and Warcraft III 
enjoy many years of popularity. It has also spawned an unintended effect that has led to something of a revolution in video gaming.

The past decade has seen a major change in video game players' expectations of newly released games. In order to achieve the pinnacle of success, new games must absolutely either include some type of level editor or must be highly customizable to cater to various users' preferences and needs. While RTS and FPS games are particularly subject to this recent requirement, the most pointed example is that of modern MMOGs. All current MMOGs, at least those that have any hope of long-term success, feature a sophisticated API (application programming interface). These APIs allow users to customize their user interface in a staggering number of ways. Figure 6.1 shows the default World of Warcraft user interface while Figure 6.2 shows a typical user modification for a player involved in end-game play that demands each member of a team know exactly what is going on with every other team member.

While it is possible for users to modify their own user interface, this is a highly complex task and requires some technical knowledge. It has become far more common for player groups to publish their tested modifications for the entire player community to use. This trend has resulted in another change in the game that is somewhat more worrisome. As players progress through the game and reach the upper levels, other players' expectations increasingly include the use of certain modifications. In fact, if a player does not have a minimal set of UI modifications installed they are frequently not allowed to join a group. This can be a serious issue if the formation of a group is necessary to achieve certain required tasks. As teams approach the most exclusive advanced content available only to the highest level players, additional requirements of specific equipment (weapons, armor, exclusive abilities, etc.) are also imposed. This exclusionary behavior closely resembles classism and is a source of frequent frustration for newer players.

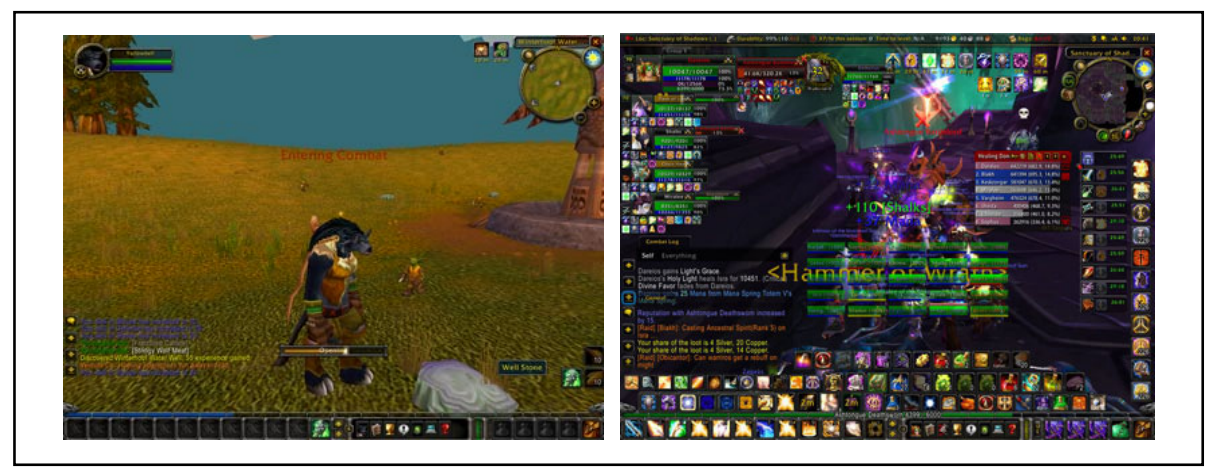

Fig. 6.1. Default WoW UI

Fig. 6.2. Modified WoW UI for team play

Elitism is a rampant problem in many MMOGs extending well beyond UI and equipment requirements. Certain combinations of avatar race and class can be highly discriminated against. For example, in World of Warcraft if you choose to be a Dwarf, a race that tends to be clumsy but very strong, and you choose to play a Rogue, a class that requires a great deal of stealth and dexterity, while being a 
perfectly valid race class combination the typical result is a high degree of ridicule by fellow players. Beyond this simple example there have been claims that the overall race design in WoW parallels post-colonialism [7]. The design choice to make Alliance races "familiar" (white Western races) versus Horde races made to be "foreign" (African, Jamaican, Native American, etc.) has caused a tremendous imbalance in the player base highly favoring the Alliance. This design decision makes salient Stuart Hall's concept of ideology and the inherent risks associated with intentional representation of social formations [10].

\section{Conclusions}

Video games have come a long way since Pong. From a simple diversion in entertainment to a dominant media market, video games have developed into a unique phenomenon that promises continued surprises and unparalleled opportunities for insight into the human psyche. As the direction of game development has been indelibly changed by the players, the player community has transformed into a distinct culture. In this transition, for instance, the distinction between an MMOG and a generic MUVE (multi-user virtual environment) has become blurred. These two very distinct genres have merged through a series of player dominated transitions reducing what was once a clear difference to a simple difference in content and AI. Now all games are, like only the MUVE was previously, virtual blank slates for players to fill with rules, norms, and traditions.

All members of the entertainment computing community must begin to understand the widespread and subtle cultural impact of what they do. Game players as well as developers of game software and those who produce the associated hardware required to play them need to have an understanding of the international scope of the issues surrounding video games. Rather than perpetuating racial stereotypes and prejudiced norms, both players and designers of game systems might use their newfound influence to begin breaking down barriers that have caused geographically separated cultures to remain distinct. It has now become possible through this subtle influence to begin laying the groundwork of the long anticipated "Utopia" of cyberspace as described so eloquently by Barlow in A Declaration of the Independence of Cyberspace [3]:

We are creating a world that all may enter without privilege or prejudice accorded by race, economic power, military force, or station of birth.

We are creating a world where anyone, anywhere may express his or her beliefs, no matter how singular, without fear of being coerced into silence or conformity.

John Perry Barlow, 1996

In the tradition of Critical Theory, it is now time to re-examine video games, the player communities, and their impact on the world outside the game. Stuart Hall advocates a return of the repressed in media studies [10]. I can not imagine a community more repressed than video gamers. By forsaking the traditional wisdom that, "video games rot your brain" and looking beyond the surface of appearances, we find that not only is there real culture happening under our proverbial noses, but we may also find 
that the attitudes, beliefs, and behaviors of the "new generation" of video gamers could very well have tremendous impact on social, political, and economic issues far beyond the boundaries of their synthetic worlds [6].

\section{References}

1. Bailenson, J.N., Blascovich, J.: Avatars. In: Encyclopedia of Human-Computer Interaction, pp. 64-68. Berkshire Publishing Group (2004)

2. Bandura, A.: Social Cognitive Theory of Mass Communication, pp. 121-154. Bryant \& Zillmann (2002)

3. Barlow, J.P.: A Declaration of the Independence of Cyberspace (1996), https://projects.eff.org/ barlow/Declaration-Final.html

4. Bartle, R.: Hearts, Clubs, Diamonds, Spades: Players Who suit MUDs (1996), http://www.mud.co.uk/richard/hcds.htm

5. Carey, J.W.: Communication As Culture, rev. edn. Routledge, New York (2009)

6. Castronova, E.: Synthetic Worlds. University of Chicago Press, Chicago (2005)

7. Corneliussen, H.G., Rettberg, J.W.: Digital Culture, Play, and Identity: A World of Warcraft Reader. MIT Press, Cambridge (2008)

8. Ducheneaut, N., Yee, N., Nickell, E., Moore, R.J.: Alone together? exploring the social dynamics of massively multiplayer online games. In: Proceedings of the SIGCHI Conference on Human Factors in Computing Systems, Montréal, Québec, Canada, April 22-27 (2006)

9. Geertz, C.: The Interpretation of Cultures. Basic Books, New York (1973)

10. Hall, S.: The Rediscovery of Ideology: The Return of the 'Repressed' in Media Studies, Michael Gurevitch et al., Culture, Society and Media, pp. 56-90. Metheun, London (1982)

11. Johnson, D.: StarCraft Fan Craft: Game Mods, Ownership, and Totally Incomplete Conversions. In: The Velvet Light Trap - Number 64, pp. 50-63 (Fall 2009)

12. Lewis, M.: Moneyball: The Art of Winning an Unfair Game. W. W. \& Company, Inc., Norton (2003)

13. Reeves, B., Malone, T.W., O'Driscoll, T.: Leadership's Online Labs. Harvard Business Review (2008)

14. Rubin, A.M.: The uses-and-gratifications perspective of media effects. In: Bryant \& Zillmann, pp. 525-548 (2002)

15. Taylor, T.L.: Play Between Worlds: Exploring Online Game Culture. MIT Press, Cambridge (2006)

16. Yee, N.: The demographics, motivations, and derived experiences of users of massively multi-user online graphical environments. Presence: Teleoper. Virtual Environ. 15(3), 309-329 (2006)

17. Yee, N.: The psychology of MMORPGs: Emotional investment, motivations, relationship formation, and problematic usage. In: Schroeder, R., Axelsson, A. (eds.) Avatars at Work and Play: Collaboration and Interaction in Shared Virtual Environments. Springer, London (2006) 